\title{
References
}

[1] R. O'N eil, Convolution operators and $L(p, q)$ spaces, Duke Math. Jour. 30 (1963), pp. 129-142.

[2] - and G. Weiss. The Hilbert transform and rearrangement of functions, Studia Math. 23 (1963), pp. 189-198.

[3] W. Orlicz, Über eine gewisse Klasse von Räumen vom Typus B. Bull. Acad. Polon. Sci. (1932) pp. 207-220.

[4] A. Zygmund, Sur les fonetions conjuguées, Fund. Math. (1929) 13, pp. 284-303.

STATE UNIVERSITY OF NEW YORK AT ALBANY

\section{Distribution function inequalities for the area integral ${ }^{*}$}

D. L. BURKH OLDER (Urbana III.) and R. F. GUNDY (New Brunswick N. J.)

Dedicated to Antoni Zygmund

Abstract. Let $A$ be the area integral of a function $u$ harmonic in the Euclidean half-space $\boldsymbol{R}^{n} \times(0, \infty)$. Information about the distribution function of a localized version of $A$ is obtained that leads to a general in egral inequality between $A$ and the nontangential maximal function of $u$ and provides a convenient approach to the study of the pointwise behavior of $u$ near the boundary. In addition, the general integral inequality of [2] between the nontangential maximal function of $u$ and that of a properly chosen conjugate is shown to hold also in the case $n>1$.

Our object here is to prove some partial distribution function inequalities for the area integral and to show how these inequalities can be used to study both the local and the global behavior of harmonic functions. Before describing our approach in detail, we consider a few of its applications.

Let $u$ be harmonic in the Euclidean half-space

$$
\boldsymbol{R}_{+}^{n+1}=\left\{(x, y): x \in \boldsymbol{R}^{n}, y>0\right\} \text {. }
$$

The area integral of $u$ is the nonnegative function $A=A_{a}(u)$ defined on $\boldsymbol{R}^{n}$ by

$$
A^{2}(x)=A_{a}^{2}(u, x)=\iint_{\Gamma(x)} y^{1-n}|\nabla u(s, y)|^{2} d s d y
$$

where $a$ is a positive real number,

$$
\Gamma(x)=\Gamma(x ; a)=\{(s, y):|x-s|<a y\},
$$

and $\nabla u=\left(\partial u / \partial y, \partial u / \partial x_{1}, \ldots, \partial u / \partial x_{n}\right)$. The nontangential maximal funetion $N=N_{a}(u)$ is defined by

$$
N(x)=\sup _{(s, y) \in \Gamma(x)}|u(s, y)|
$$

* This research was supported in part by the National Science Foundation under grants GP 28154 and GP 19222. We are also indebted to the Institut MittagLeffler and our celleagues there during the summer of 1971 for the excellent environment in which this work was carried out. In particular, we thank Charles Fefferman for many conversations on questions related to those discussed here. 
Now let $\Phi$ be any function on $[0, \infty]$ such that $0<\Phi(1)<\infty$ and

$$
\Phi(b)=\int_{0}^{b} \varphi(\lambda) d \lambda, \quad 0 \leqslant b \leqslant \infty,
$$

for some nonnegative measurable function $\varphi$ on $(0, \infty)$ satisfying the growth condition

$$
\varphi(2 \lambda) \leqslant c \varphi(\lambda), \quad \lambda>0 .
$$

Throughout the paper, the letter $c$ denotes a positive real number, not always the same number in different lines. The growth condition on $\varphi$ implies a comparable one on $\Phi$ and so $\Phi(\lambda)$ is finite and positive for all finite and positive $\lambda$. If $0<p<\infty$, then $\Phi(b)=b^{p}, \Phi(b)=\log (b+1)$, and $\Phi(b)=(b+1) \log (b+1)$ are examples of such functions. The following theorem, one of our main results, shows that $A$ and $N$ are remarkably closely related.

THEOREM 1. Under the above conditions

$$
\int_{\boldsymbol{R}^{n}} \Phi(A) d x \leqslant e \int_{\boldsymbol{R}^{n}} \Phi(N) d x .
$$

If the left side of $(3)$ is finite, then $\lim u(x, y)$ exists, and is finite and constant, for $x \in \boldsymbol{R}^{n}$. If $u$ is normalized so that this limit is zero, then the converse inequality holds:

$$
\int_{\boldsymbol{R}^{n}} \Phi(N) d x \leqslant 0 \int_{\boldsymbol{R}^{n}} \Phi(A) d x .
$$

The choice of $c_{(3)}$ and $e_{(4)}$ depends only on $n, a$, and the growth constant $c_{(2)}$. The addition of a constant to $u$ does not change $A$ but ordinarily does change $N$; normalization is necessary to assure (4).

Theorem 1 can be used to prove inequalities of the form

$$
\int_{\boldsymbol{R}^{n}} \Phi(N(v)) d x \leqslant c \int_{\boldsymbol{R}^{n}} \Phi(N(u)) d x
$$

where $v$, normalized to vanish at $y=\infty$, is a conjugate of $u$ in a generalized sense ([8], [15]). For the case $n=1,(5)$ is already known [2]. Here is another proof: For $n=1, v$ is conjugate to $u$ if $u+i v$ is analytic in the upper half-plane. The Cauchy-Riemann equations imply that $|\nabla v|$ $=|\nabla u|$, hence $A(v)=A(u)$. By applying (3) to $N(u),(4)$ to $N(v)$, and using $A(v)=A(u)$, we obtain (5). The case $n>1$ is discussed in Section 4 .

Bounds on the $L^{p}$ norm of the area integral have been given by many authors. We mention especially the work, for the case $n=1$, of Marcinkiewicz and Zygmund [9] and Calderón [5], and the work, for the case $n>1$, of Stein [12], [14], Gasper [7], and Segovia [10]. Recently, Feffer- man and Stein [6] obtained Theorem 1 for several special cases including $\Phi$ a power: $\Phi(b)=p(0<p<\infty)$. They also proved

$$
\|A\|_{p} \leqslant c_{p, n, a}\left\|N_{0}\right\|_{p}, \quad 0<p<\infty,
$$

where $N_{0}$ is the radial maximal function:

$$
N_{0}(x)=\sup _{y>0}|u(x, y)|
$$

It is interesting to note that if $N$ is replaced by $N_{0}$ in (3), then (3) no longer holds for all $\Phi$ satisfying the requirements of Theorem 1 ; see [2], p. 152 .

The $\Phi$ inequalities of Theorem 1 follow easily from the partial distribution function inequalities proved below. The latter also provide at convenient and illuminating approach to some of the results on the local behavior of harmonic functions due to Marcinkiewicz and Zygmund [9], Spencer [11], Calderón [3], [4], and Stein [13]. As an example, the following result of Calderón [3] can be mentioned: If $u$ is nontangentially bounded at every point of a measurable set $E \subset \boldsymbol{R}^{n}$, then $u$ has a nontangential limit at almost every point of $E$. We return to these questions in Section 3.

1. The basic inequalities. Keeping the notation already established, we consider local versions of the area integral and the nontangential maximal function. Let $R$ be a measurable subset of $\boldsymbol{R}_{+}^{n+1}$ and $A_{R}$ the nonnegative function on $\boldsymbol{R}^{n}$ defined by

$$
A_{R}^{2}(x)=\iint_{\Gamma(x) \cap R} y^{1-n}|\nabla u(s, y)|^{2} d s d y .
$$

If $\Gamma(x) \cap R$ is nonempty, let

$$
\begin{gathered}
N_{R}(x)=\sup _{(s, y) \in \Gamma(x) \cap R}|u(s, y)|, \\
D_{R}(x)=\sup _{(s, y) \in \Gamma(x) \cap R} y|\nabla u(s, y)| ;
\end{gathered}
$$

otherwise, let $N_{R}(x)=D_{R}(x)=0$. In the following theorem, $m\left(A_{R}>\lambda\right)$ denotes the Lebesgue measure of the set of $x \in \boldsymbol{R}^{n}$ satisfying $A_{R}(x)>\lambda$.

THEOREM 2. Let $G$ be a bounded open subset of $\boldsymbol{R}^{n}$ and $R$ the interior of the complement of $\bigcup_{x \in G} \Gamma(x)$. Let $\alpha>1$ and $\beta>1$. Then

$$
m\left(A_{R}>\lambda\right) \leqslant c m\left(c N_{R}>\lambda\right)+c m\left(c D_{R}>\lambda\right)
$$

for all $\lambda>0$ satisfying

$$
m\left(A_{R}>\lambda\right)<\alpha m\left(A_{R}>\beta \lambda\right) .
$$

The ehoice of $c$ depends only on $\alpha, \beta, n$ and $a$. 
In the proof, only the harmonicity of $u$ in $R$ is used; $u$ need not be defined outside of $R$. Note that over each component of $G$ the shape of $R$ resembles that of a mountain range. The theorem is also true with $G$ unbounded; this follows easily from the bounded case since $A_{R}, N_{R}$, and $D_{R}$ increase as $R$ increases.

In the following theorem, we need to use an interesting variant of the nontangential maximal function. If $\Gamma(x) \cap R$ is empty, let $N_{R}^{0}(x)=0$; otherwise, let

$$
N_{R}^{0}(x)=\sup _{(s, y) \in \Gamma(x) \cap R}\left|u(s, y)-u\left(s, y_{s}\right)\right|
$$

where $\left(s, y_{s}\right)$ is the point on the upper boundary of $R$ directly above $(s, y)$ :

$$
y_{s}=\sup \{y:(s, y) \in R\}
$$

THeOREM 3. Let $G$ be a bounded open subset of $\boldsymbol{R}^{n}$ and $R$ the interior of the complement of $\bigcup_{x \neq G} \Gamma(x)$. Let $\alpha>1$ and $\beta>1$. Then

$$
m\left(N_{R}^{0}>\lambda\right) \leqslant c m\left(c A_{R}>\lambda\right)+c m\left(c D_{R}>\lambda\right)
$$

for all $\lambda>0$ satisfying

$$
m\left(N_{R}^{0}>\lambda\right)<\alpha m\left(N_{R}^{0}>\beta \lambda\right) .
$$

The choice of $c$ depends onty on $\alpha, \beta, n$ and $a$.

We now use additional variants of $N$ and $A$ to simplify the right sides of (7) and (9). Consider the truncated cones

$$
\Gamma(x ; b, k)=\{(s, y):|x-s|<b y, 0<y<k\}
$$

where $b$ and $k$ are positive real numbers. Let $N_{b, k}$ be defined by (1) with $\Gamma(x)$ replaced by $\Gamma(x ; b, k)$. Define $A_{b, k}$ and $D_{b, k}$ analogously.

LEMNA 1. Let $G$ and $R$ be as in Theorems 2 and 3 and let $k$ be a positive number such that ak is not less than the diameter of $G$. Let $b=2 a$. Then

$$
\begin{aligned}
& D_{R} \leqslant c N_{b, k}, \\
& D_{R} \leqslant c A_{b, k},
\end{aligned}
$$

and the choice of $c$ depends only on $n$ and a. Therefore, in Theorem 2, (7) can be replaced by

$$
m\left(A_{R}>\lambda\right) \leqslant c m\left(c N_{b, k}>\lambda, G\right)
$$

and, in Theorem 3, (9) can be replaced by

$$
m\left(N_{R}^{0}>\lambda\right) \leqslant c m\left(c A_{b, k}>\lambda, G\right)
$$

Note that the comma is sometimes used to denote intersection.

Proof. The height of $R$ does not exceed $h=\frac{1}{2} k$ : if $(x, y) \epsilon R$, then

$$
a y \leqslant \inf \{|x-s|: s \notin G\} \leqslant a h .
$$

Therefore, $D_{R} \leqslant D_{a, h}$. By Lemmas 4 and 5 of Stein [13], $D_{a, h} \leqslant c N_{b, k}$ and $D_{a, h} \leqslant c A_{b, k}$. Accordingly, (11) and (12) follow.

Because both $N_{R}$ and $D_{R}$ vanish off $G$ and are dominated by $c N_{b, k}$, the right side of (7) is dominated by the right side of $\left(7^{\prime}\right)$. A similar comparison holds for $(9)$ and $\left(9^{\prime}\right)$. Therefore, the last statement of Lemma 1 follows from (7) and (9).

Here is another basic inequality.

LemMa 2. Let $b>a>0$. Then, for all $\lambda>0$,

$$
m\left(N_{b}>\lambda\right) \leqslant c m\left(N_{a}>\lambda\right) \text {. }
$$

The choice of $c$ depends only on $n$ and the ratio $a / b$.

Note that (13) implies

$$
m\left(N_{b, k}>\lambda, G\right) \leqslant m\left(N_{b}>\lambda\right) \leqslant c m\left(N_{a}>\lambda\right) .
$$

Furthermore, if $\Phi$ is as in Theorem 1 , then

$$
\int_{\mathbf{R}^{n}} \Phi\left(N_{b}\right) d x \leqslant e \int_{\boldsymbol{R}^{n}} \Phi\left(N_{a}\right) d x
$$

To see this, use Fubini's theorem to obtain

$$
\int_{\boldsymbol{R}^{n}} \Phi\left(N_{b}\right) d x=\int_{0}^{\infty} \varphi(\lambda) m\left(N_{b}>\lambda\right) d \lambda
$$

then use (13). Note that $c_{(15)}=c_{(13)}$.

Proof. Let $B(x, y)=\left\{s \in \boldsymbol{R}^{n}:|x-s|<y\right\}$. Then $B(x, a y) \subset\left\{N_{a}>\lambda\right\}$ for all $(x, y) \epsilon \boldsymbol{R}_{+}^{n+1}$ satisfying $|u(x, y)|>\lambda$. In fact,

$$
\left\{N_{a}>\lambda\right\}=\bigcup\{B(x, a y):|u(x, y)|>\lambda\} .
$$

Let $f$ be the characteristic function of this set and $f^{*}$ the maximal function of $f$ defined by

Then

$$
f^{*}(x)=\sup _{y>0} \int_{B(x, y)} f(s) d s / m(B(x, y))
$$

$$
\left\{N_{b}>\lambda\right\} \subset\left\{f^{*} \geqslant a\right\}
$$

where $\alpha=a^{n} /(a+b)^{n}$. For, if $\dot{N}_{b}(x)>\lambda$, then there is a point $(s, y)$ satisfying $|u(s, y)|>\lambda$ and $x \in B(s, b y)$. Note that $f=1$ on $B(s, a y)$ since $B(s, a y)$ $\subset\left\{N_{a}>\lambda\right\}$. Therefore,

$$
\begin{aligned}
f^{*}(x) & \geqslant m(B(s, a y) \cap B(x, a y+b y)) / m(B(x, a y+b y)) \\
& =m(B(s, a y)) / m(B(x, a y+b y))=\alpha,
\end{aligned}
$$


which proves (16). Using (16) and a variant of the Hardy-Littlewood maximal theorem (see, for example, Chapter I of [14]), we have

$$
a m\left(N_{b}>\lambda\right) \leqslant \alpha m\left(f^{*} \geqslant \alpha\right) \leqslant c\|f\|_{1}=a m\left(N_{a}>\lambda\right)
$$

with the choice of $\theta$ depending only on $n$. This completes the proof of Lemma 2.

As we have seen above, any "complete" distribution function inequality, such as (13), implies a corresponding $\Phi$ inequality. However, "partial" distribution function inequalities, such as those contained in Theorems 2 and 3 , can also be used to obtain $\Phi$ inequalities as we now show.

2. Proof of Theorem 1. In addition to Theorems 2 and 3 , which we prove below, we need the following elementary lemma.

LeMna 3. Let $f: \boldsymbol{R}^{n} \rightarrow[0, \infty]$ be measurable with compact support. Let $\Phi$ be as in Theorem 1 and suppose that $\alpha>1, \beta>1,0<\gamma<\alpha / \beta$, and

Then

$$
\varphi(\beta \lambda) \leqslant \gamma \varphi(\lambda), \quad \lambda>0 .
$$

where

$$
\int_{\boldsymbol{R}^{n}} \Phi(f) d x \leqslant \frac{\alpha \beta \gamma}{\alpha-\beta \gamma} \int_{\Lambda} \varphi(\lambda) m(f>\lambda) d \lambda
$$

$$
\Lambda=\{\lambda>0: m(f>\lambda)<a m(f>\beta \lambda)\} .
$$

The easy proof is essentially contained in [1] and is omitted.

To prove (3), we apply the lemma to $f=A_{R}$ for $R$ and $G$ as in Theorem 2. Notice that $A_{R}$ vanishes outside of $G$. Let $\beta=2, \gamma=c_{(2)}$, and $\alpha=4 \gamma$. Then, by Lemma 3 ,

$$
\int_{\mathbb{R}^{n}} \Phi\left(A_{R}\right) d x \leqslant \alpha \int_{\Lambda} \varphi(\lambda) m\left(A_{R}>\lambda\right) d \lambda
$$

where $A$ is the set of all $\lambda>0$ satisfying (8). By $\left(7^{\prime}\right)$ and (14), the right side is no greater than

$$
a \int_{0}^{\infty} \varphi(\lambda) c m(c N>\lambda) d \lambda=c \alpha \int_{\boldsymbol{R}^{n}} \Phi(c N) d x \leqslant c \int_{\mathbf{R}^{n}} \Phi(N) d x .
$$

Therefore, (3) holds with $A$ replaced by $A_{R}$. Now let $R \uparrow \boldsymbol{R}_{+}^{n+1}$. By the monotone convergence theorem, (3) follows.

We now consider the converse inequality and let $b=2 a$. Using the same pattern of reasoning as above, here in conjunction with Theorem 3, we obtain

$$
\int_{\mathbf{R}^{n}} \Phi\left(N_{R}^{0}\right) d x \leqslant c \int_{\mathbf{R}^{n}} \Phi\left(A_{b}\right) d x
$$

We assume from now on that the right side of (17) is finite and show first that $u(0, y)$ converges as $y \rightarrow \infty$. We restrict our attention to the regions $R=R_{t}$ corresponding to $G=B(0, a t)=\{x:|x|<a t\}$. Suppose that $x$ is any point in $\boldsymbol{R}^{n}$ and that $|x|<a y<a z<a t$. Then $(0, y)$ and $(0, z)$ belong to $\Gamma(x) \cap R$ and it follows from the definition of $N_{R}^{0}$ that

$$
|u(0, y)-u(0, z)| \leqslant|u(0, y)-u(0, t)|+|u(0, z)-u(0, t)| \leqslant 2 N_{R}^{0}(x) .
$$

Therefore,

$$
\delta=\frac{1}{3} \limsup _{y, z \rightarrow \infty}|u(0, y)-u(0, z)| \leqslant \liminf _{t \rightarrow \infty} N_{R}^{0}(x)
$$

and, by Fatou's lemma and (17), we have

$$
\int_{\mathbf{R}^{n}} \Phi(\delta) d x \leqslant c \int_{\boldsymbol{R}^{n}} \Phi\left(A_{b}\right) d x<\infty
$$

which gives $\Phi(\delta)=0$. Therefore, $\delta=0$ and this implies that $u(0, y)$ converges as $y \rightarrow \infty$.

Using the mean value theorem and (12), we have that

$$
|u(s, y)-u(0, y)| \leqslant \sup _{t>0} D_{R}\left(x_{0}\right)|s| y^{-1} \leqslant c A_{b}\left(x_{0}\right)|s| y^{-1}
$$

provided $\left|x_{0}-s\right|<a y$ and $\left|x_{0}\right|<a y$. Since $A_{b}\left(x_{0}\right)$ is finite for at least one $x_{0}$,

$$
\left.\lim _{y \rightarrow \infty} \mid u(s, y) \rightarrow u(0, y)\right\}=0,
$$

and the convergence is uniform for $s \in B(0, r)$. This proves the existence, finiteness, and constancy of the limit of $u(\cdot, y)$ as $y \rightarrow \infty$. From now on, assume this limit is 0 .

Let

$$
\begin{aligned}
f_{r, R}(x) & =\sup \left\{\left|u(s, y)-u\left(s, y_{s}\right)\right|:(s, y) \epsilon \Gamma(x) \cap R,|s|<r\right\}, \\
f_{r}(x) & =\sup \{|u(s, y)|:(s, y) \epsilon \Gamma(x),|s|<r\} .
\end{aligned}
$$

As usual, if the sets are empty, $f_{r, R}(x)=f_{r}(x)=0$. Then $f_{r, R} \leqslant N_{R}^{0}$ and

$$
\lim _{t \rightarrow \infty} f_{r, R}=f_{r}, \lim _{r \rightarrow \infty} f_{r}=N \text {. }
$$

Using (17) and Fatou's lemma, we obtain

$$
\int_{\boldsymbol{R}^{n}} \Phi(N) d x \leqslant \epsilon \int_{\boldsymbol{R}^{n}} \Phi\left(A_{b}\right) d x
$$

In view of (10̃), this inequality also holds with $N$ replaced by $N_{b}$. This completes the proof of Theorem 1 . 
3. Local behavior of harmonic functions. Our aim here is to illustrate how partial distribution function inequalities can be used to study problems of local behavior. First, consider the following result of Calderón [4]: If $u$ is nontangentially bounded at every point of a measurable set $E$, then $A_{a, k}$ is finite almost everywhere on $E$. The first step of the proof is to show that

$$
E \underset{\text { a.e. }}{\subset}\left\{N_{b, k}<\infty\right\} \text {. }
$$

This is not difficult and rests on a familiar point of density argument (see Calderón [4]). The key step is to show that

$$
\left\{N_{b, k}<\infty\right\} \underset{\text { a.e. }}{\subset}\left\{A_{a, k}<\infty\right\} .
$$

We need to do this only for $b=2 a$. If (18) does not hold, then

$$
m\left(A_{\alpha, k}=\infty, c N_{b, k} \leqslant \lambda\right)>0,
$$

for some $\lambda>0$, with $c$ the constant appearing in $\left(7^{\prime}\right)$. Let $E_{0}$ be a measurable subset of the set in (19) with diameter not greater than $\frac{1}{2} a k$ and such that $m\left(E_{0}\right)>0$. Let $G_{j}$ be an open set containing $E_{0}$ with diameter not greater than $a k$ and such that $m\left(G_{j}-E_{0}\right) \rightarrow 0$ as $j \rightarrow \infty$. Let $R_{j}$ be the region; defined in Theorem 2 , corresponding to $G_{j}$. Note that $A_{R_{j}}(x)=\infty$ for $x \in E_{0}$. Therefore,

$$
m\left(E_{0}\right) \leqslant m\left(A_{R_{j}}>\beta \lambda\right) \leqslant m\left(A_{R_{j}}>\lambda\right) \leqslant m\left(G_{j}\right)
$$

and, since $m\left(G_{j}\right) \rightarrow m\left(E_{0}\right)$, the number $\lambda$ satisfies (8) for all large $j$. So, for such $j$, we can apply Theorem 2 and Lemma 1 to obtain

$$
m\left(A_{R_{j}}>\lambda\right) \leqslant c m\left(c N_{b, k}>\lambda, G_{j}\right) .
$$

But the right side converges to $c m\left(c N_{b, k}>\lambda, E_{0}\right)=0$ while the left side converges to $m\left(E_{0}\right)$. Accordingly, (19) cannot hold and the proof of the key step is complete.

Now consider Stein's result in the opposite direction (see [13], which also contains some remarks about the historical background): If $A_{b, k}$ is finite at every point of a measurable set $E$, then $u$ has a nontangential limit at almost every point of $E$. Let $\varepsilon>0$. We denote by $I I$ the set of all $x \in \boldsymbol{R}^{n}$ such that either

or

$$
\lim \sup u(s, y)-\liminf u(s, y)>\varepsilon
$$

$$
\limsup |u(s, y)|=\infty
$$

as $(s, y) \rightarrow(x, 0),(s, y) \in \Gamma(x ; a, k)$. Stein's result is a simple consequence of the fact that

(20)

$$
m(E \cap M)=0
$$

for all $\varepsilon>0, a>0$.
We show below that (20) holds for all $\varepsilon>0$ with $a=\frac{1}{2} b$. This is enough! The special case implies that $u$ is nontangentially bounded almost everywhere on $E$. By Calderón's result, we have that

$$
E \underset{\text { a.e. }}{\subset} \bigcup_{b>0}\left\{A_{b, k}<\infty\right\}
$$

Therefore, by the special case, we have that (20) holds for all $a$.

Now fix $\varepsilon>0$ and let $a=\frac{1}{2} b$. Let $\beta>1$ as in Theorem 3 and choose $\lambda$ to satisfy $2 \beta \lambda=\varepsilon$. If $(20)$ does not hold, then $m(E \cap M)>0$ and, for $h$ sufficiently small,

$$
m\left(c A_{b, h} \leqslant \lambda, M\right)>0 .
$$

Here we have used the fact that $A_{b, h}(x) \rightarrow 0$ as $h \rightarrow 0$ for all $x \in E$. Choose $c$ to be the constant appearing in $\left(9^{\prime}\right)$. Let $E_{0}$ be a measurable subset of the set in (21) with diameter not greater than $\frac{1}{2} a h$ and such that $m\left(E_{0}\right)>0$. Let $G_{j}$ be an open set containing $E_{0}$ with diameter not greater than $a h$ and such that $m\left(G_{j}-E_{0}\right) \rightarrow 0$ as $j \rightarrow \infty$. Let $R_{j}$ be the region, defined in Theorem 3, corresponding to $G_{j}$. Let $x \in E_{0}$. Using the definition of $M$ and $\lambda$, the continuity of $u$ in a neighborhood of $\left(x, y_{x}\right)$, and the fact that $\left|y_{s}-y_{x}\right| \leqslant a^{-1}|s-x|$, we obtain $N_{R_{j}}^{0}(x)>\beta \lambda$. Therefore,

$$
m\left(E_{0}\right) \leqslant m\left(N_{R_{j}}^{0}>\beta \lambda\right) \leqslant m\left(N_{R_{j}}^{0}>\lambda\right) \leqslant m\left(G_{j}\right)
$$

and $\lambda$ satisfies (10) for all large $j$. Applying Theorem 3 and Lemma 1 , we have

$m\left(E_{0}\right)=\lim _{j \rightarrow \infty} m\left(N_{R_{j}}^{0}>\lambda\right) \leqslant \lim _{j \rightarrow \infty} c m\left(c A_{b, h}>\lambda, G_{j}\right)=c m\left(c A_{b, h}>\lambda, E_{0}\right)=0$,

a contradiction. This completes the proof of Stein's result.

4. Conjugate harmonic functions. Suppose $u, v_{1}, v_{2}, \ldots, v_{n}$ are harmonic in $\boldsymbol{R}_{+}^{n+1}$ and satisfy the generalized Cauchy-Riemann equations:

$$
\begin{gathered}
\frac{\partial u}{\partial y}+\frac{\partial v_{1}}{\partial x_{1}}+\ldots+\frac{\partial v_{n}}{\partial x_{n}}=0 \\
\frac{\partial u}{\partial x_{k}}=\frac{\partial v_{k}}{\partial y}, \frac{\partial v_{k}}{\partial x_{j}}=\frac{\partial v_{j}}{\partial x_{k}}, \quad j, k=1, \ldots, n
\end{gathered}
$$

Stein and Weiss [15] and Stein [13], [14] have made a deep study of such systems of conjugate harmonic functions. Here let $v=\left(v_{1}, \ldots, v_{n}\right)$,

$$
|v|=\left(\sum_{k=1}^{n} v_{k}^{2}\right)^{\frac{1}{2}},
$$

and define $N(v)$ by $(1)$ with $u$ replaced by $v$. Then we have the following generalization of Theorem 2 of [2]: 
Theorem 4. Let $u$ be harmonic in $\boldsymbol{R}_{+}^{n+1}$, let $\Phi$ be as in Theorem 1 , and suppose the right side of (22), below, is finite. Then there is a $v=\left(v_{1}, \ldots, v_{n}\right)$, conjugate to $u$ in the above sense, such that

$$
\int_{\boldsymbol{R}^{n}} \Phi(N(v)) d x \leqslant c \int_{\boldsymbol{R}^{n}} \Phi(N(u)) d x .
$$

The choice of $c$ depends only on $n, a$, and the growth constant of $\varphi$.

Proof. There is a $v$, conjugate to $u$, such that $v$ vanishes at $y=\infty$ :

$$
\lim _{y \rightarrow \infty} v(x, y)=0 \text {. }
$$

For the case $n=1$, the proof of this is contained in [2]; the proof of the general case is similar with Riesz transforms playing the role of the Hilbert transform. Now (23) implies that $\partial v_{k} / \partial x_{j}$ vanishes at $y=\infty$; using this, we obtain

$$
\iint_{\Gamma(x ; a)} y^{1-n}\left|\frac{\partial v_{k}}{\partial x_{j}}\right|^{2} d s d y \leqslant c \iint_{\Gamma(x ; b)} y^{1-n}\left|\frac{\partial v_{k}}{\partial y}\right|^{2} d s d y
$$

by slightly modifying the proof of Stein's Lemma 2.5.1 ([14], p. 213). Here $b=2 a$. Therefore, using $\partial v_{k} / \partial y=\partial u / \partial x_{k}$, we have

Hence, by Theorem 1 and (15),

$$
\begin{aligned}
\int_{\mathbf{R}^{n}} \Phi\left(N\left(v_{k}\right)\right) d x & \leqslant c \int_{\mathbf{R}^{n}} \Phi\left(A\left(v_{k}\right)\right) d x \leqslant c \int_{\boldsymbol{R}^{n}} \Phi\left(A_{b}(u)\right) d x \\
& \leqslant c \int_{\boldsymbol{R}^{n}} \Phi\left(N_{b}(u)\right) d x \leqslant c \int_{\boldsymbol{R}^{n}} \Phi(N(u)) d x .
\end{aligned}
$$

Theorem 4 now follows with the use of the following elementary facts:

$$
\begin{gathered}
N(v) \leqslant \sum_{k=1}^{n} N\left(v_{k}\right), \\
\Phi\left(\sum_{k=1}^{n} \lambda_{k}\right) \leqslant \sum_{k=1}^{n} \Phi\left(2^{n} \lambda_{k}\right) \leqslant c \sum_{k=1}^{n} \Phi\left(\lambda_{k}\right), \quad \lambda_{k} \geqslant 0, k=1, \ldots, n .
\end{gathered}
$$

5. Proof of Theorem 2. We need the following lemma.

LEMMa 4. Suppose that $G$ is an open bounded nonempty subset of $\boldsymbol{R}^{n}$ and that $F$ is its complement. Let $\alpha>1$ and suppose that $E$ is a measurable subset of $G$ satisfying $m(G) \leqslant \alpha m(E)$. Then there is a ball $B \subset G$, with at loast one of its boundary points in $F$, such that

$$
m(B) \leqslant \operatorname{cam}(E \cap B) \text {. }
$$

The choice of $c$ depends only on $n$.
Proof. There is a Whitney cube $Q \subset G$ (for example, see [14], p. 16) such that

$$
\begin{gathered}
m(Q) \leqslant \alpha m(E \cap Q), \\
q \leqslant d \leqslant 4 q,
\end{gathered}
$$

where $q$ is the diameter of $Q$ and $d$ is the distance from $Q$ to $F$. Now consider all balls centered at the center of $Q$, containing $Q$, and contained in $G$. By the left side of (27), this family is nonempty; let $B$ denote its union. The maximality of $B$ in the above family assures that at least one of its boundary points is in $F$. By (26) and the right side of (27), we obtain (25).

We now turn to the proof of Theorem 2. For the sake of simplicity and with no essential loss of generality, we assume that $a=1$.

Suppose that $\lambda$ satisfies (8). Let $R_{\varepsilon}=\{(x, y) \in R: y>\varepsilon\}$ for $\varepsilon>0$. Since $A_{R_{\varepsilon}} \rightarrow A_{R}$ as $\varepsilon \rightarrow 0$, the inequality

$$
m\left(A_{R_{\varepsilon}}>\lambda\right)<\alpha m\left(A_{R_{\varepsilon}}>\beta \lambda\right)
$$

holds for all small $\varepsilon$. As we now show, this inequality implies that (7) holds with $R$ replaced by $R_{\varepsilon}$. The result for $R$ follows by letting $\varepsilon \rightarrow 0$.

Note that $A_{R_{\varepsilon}}$ is a continuous function vanishing outside of $G$. Therefore, $G_{0}=\left\{A_{R_{\varepsilon}}>\lambda\right\}$ is an open set whose closure is contained in $G$. Let

$$
E=\left\{A_{R_{\varepsilon}} \geqslant \beta \lambda, N_{R_{\varepsilon}} \leqslant \gamma \lambda, D_{R_{\varepsilon}} \leqslant \delta \lambda\right\}
$$

where $\gamma$ and $\delta$ are positive numbers to be chosen later. Assume that (28) holds. Then

$$
m\left(G_{0}\right) \leqslant a m(E)+a m\left(N_{R_{\varepsilon}}>\gamma \lambda\right)+a m\left(D_{R_{\varepsilon}}>\delta \lambda\right) .
$$

The key step of the proof is to show that

$$
\alpha m(E) \leqslant \frac{1}{2} m\left(G_{0}\right)
$$

provided $\gamma$ and $\delta$ are suitably chosen, the choice to depend only on $\alpha, \beta$, and $n$. The desired inequality follows:

$$
m\left(G_{0}\right) \leqslant 2 \alpha m\left(N_{R_{\varepsilon}}>\gamma \lambda\right)+2 \alpha m\left(D_{R_{\varepsilon}}>\delta \lambda\right) .
$$

Suppose that (29) does not hold. Then $m\left(G_{0}\right)<2 \alpha m(E)$ and, by Lemma 4 , there is a ball $B \subset G_{0}$, with at least one of its boundary points not in $G_{0}$, such that

$$
m(B) \leqslant \alpha_{0} m(E \cap B),
$$

where $\alpha_{0}=2 \alpha c_{(25)}$. Without loss of generality, assume that $B$ is centered at the origin and has unit radius. Let $V$ be the interior of the cone with base $B$ and vertex at $(0,1)$. Then the closure of $V_{\varepsilon}=\{(x, y) \in V: y>\varepsilon\}$ is contained in $R$. Choose $0<\eta<\frac{1}{2}$ so that the ball $B_{0}$ with center at 
the origin and radius $1-2 \eta$ satisfies $m\left(B_{0}\right) / m(B)=1-\left(2 \alpha_{0}\right)^{-1}$. Then, letting $E_{0}=E \cap B_{0}$, we have that

(30)

$$
m(B) \leqslant 2 \alpha_{0} m\left(E_{0}\right) .
$$

Finally, let

$$
W=\bigcup_{x \in E_{0}} \Gamma(x) \cap V_{\varepsilon}
$$

Note that $W \subset V_{\varepsilon}$ and, except for its more "mountainous" lower boundary, $W$ has roughly the same basic shape as $V_{\varepsilon}$. Certainly, $W$ is a Lipschitz domain. Observe that

$$
|u(s, y)| \leqslant \gamma \lambda, \quad y|\nabla u(s, y)| \leqslant \delta \lambda
$$

for all $(s, y) \in W$, hence for all $(s, y) \epsilon \partial W$; indeed, these inequalities hold for all $(s, y)$ in $\Gamma(x) \cap R_{\varepsilon}$ if $x \in E_{0}$.

Now consider the area integral relative to the domain $W$; see (6). For a suitable $\delta$, the choice of which depends only on $a, \beta$, and $n$, we have that

$$
A_{W}^{2}(x) \geqslant \frac{1}{2}\left(\beta^{2}-1\right) \lambda^{2}, \quad x \in E_{0} .
$$

To prove this, we fix $x \in E_{0}$ and observe first that

where

$$
\beta^{2} \lambda^{2} \leqslant A_{R_{\varepsilon}}^{2}(x)=A_{W}^{2}(x)+A_{U_{1}}^{2}(x)+A_{U_{2}}^{2}(x)+A_{U_{3}}^{2}(x)
$$

$$
\begin{aligned}
& \bar{J}_{1}=\left\{(s, y) \epsilon R_{\varepsilon}:|s|<y-1, y>1\right\}, \\
& \bar{J}_{2}=\left\{(s, y) \epsilon \Gamma(x) \cap R_{\varepsilon}:|s|>y-1, y>1\right\}, \\
& \bar{J}_{3}=\left\{(s, y) \epsilon \Gamma(x) \cap R_{\varepsilon}:(s, y) \notin W, y<1\right\} .
\end{aligned}
$$

Using now the fact that $B$ has a boundary point, say $x_{0}$, not in $G_{0}$, we obtain $U_{1} \subset \Gamma\left(x_{0}\right) \cap R_{\varepsilon}$ and

$$
A_{U_{1}}^{2}(x) \leqslant A_{R_{\varepsilon}}^{2}\left(x_{0}\right) \leqslant \lambda^{2} .
$$

Let $v_{n}$ denote the volume of a ball of unit radius in $\boldsymbol{R}^{n}$. Then, by (31),

$$
\begin{aligned}
A_{U_{2}}^{2}(x) & =\iint_{T(x) \cap U_{2}} y^{1-n}|\nabla u(s, y)|^{2} d s d y \leqslant \int_{1}^{\infty} \int_{\substack{|x-s|<y \\
|s|>y-1}} y^{1-n} \delta^{2} \lambda^{2} y^{-2} d s d y \\
& =\delta^{2} \lambda^{2} \int_{1}^{\infty}\left[v_{n} y^{n}-v_{n}(y-1)^{n}\right] y^{-n-1} d y \leqslant v_{n} 2^{n} \delta^{2} \lambda^{2} \int_{1}^{\infty} y^{-2} d y=e \delta^{2} \lambda^{2} .
\end{aligned}
$$

We now use the fact that $x \in B_{0}$. This means that $|x|<1-2 \eta$ and if $(s, y) \in U_{3}$, then $\eta<y<1$. Therefore,

$$
\boldsymbol{A}_{U_{\mathrm{a}}}^{2}(x) \leqslant \int_{\eta}^{1} \int_{|s|<y+1} y^{1-n} \delta^{2} \lambda^{2} y^{-2} d s d y=c \delta^{2} \lambda^{2}
$$

These estimates imply the statement containing (32).
We now use Green's theorem assuming that the above region $W$ is smooth enough for the theorem to apply. Whether or not this assumption is generally valid, $W$ can always be approximated by a smoother region (see [13], [14]) to achieve the same end.

Let $\sigma$ denote the measure of the surface area of $W$ and let $\partial / \partial n$ denote the directional derivative along the inward normal. Then $\sigma(\partial W)$ $\leqslant 4 m(B)$ since $|\partial y| \partial n \mid \geqslant 2^{-k}$ almost everywhere with respect to $\sigma$. Now using (32), (31), (30), and the identity $\Delta u^{2}=2|\nabla u|^{2}$, we have that

$$
\begin{aligned}
\left(\beta^{2}-1\right) \lambda^{2} m\left(E_{0}\right) & \leqslant 2 \int_{\mathbf{R}^{n}} A_{W}^{2}(x) d x=v_{n} \int_{W} y \Delta u^{2} d s d y \\
& =v_{n} \int_{\partial W} u^{2} \frac{\partial y}{\partial n} d \sigma-2 v_{n} \int_{\partial W} y \frac{\partial u}{\partial n} u d \sigma \\
& \leqslant v_{n} \gamma^{2} \lambda^{2} \sigma(\partial W)+2 v_{n} \gamma \delta \lambda^{2} \sigma(\partial W) \\
& \leqslant 4 v_{n}\left(\gamma^{2}+2 \gamma \delta\right) \lambda^{2} m(B) \\
& \leqslant 8 a_{0} v_{n}\left(\gamma^{2}+2 \gamma \delta\right) \lambda^{2} m\left(E_{0}\right) .
\end{aligned}
$$

This gives a contradiction for $\gamma$ suitably small. This completes the proof of (29).

6. Proof of Theorem 3. Again, we set $a=1$ and let $R_{\varepsilon}=\{(x, y) \in R$ : $y>\varepsilon\}$ for $\varepsilon>0$. Suppose that $\lambda$ satisfies (10). Since $N_{R_{\varepsilon}}^{0} \uparrow N_{R}^{0}$ as $\varepsilon \downarrow 0$, the inequality

$$
m\left(N_{R_{\varepsilon}}^{0}>\lambda\right)<\alpha m\left(N_{R_{\varepsilon}}^{0}>\beta \lambda\right)
$$

holds for all small $\varepsilon$. This inequality implies that (9) holds with $R$ replaced by $R_{\varepsilon}$ as we now show. The result for $R$ follows by letting $\varepsilon \rightarrow 0$.

If $x$ is a point of $G$ within $\delta$ of the boundary of $G$, then $\left|y-y_{s}\right| \leqslant \delta$ for $(s, y) \epsilon \Gamma(x) \cap R_{\varepsilon}$. By the uniform continuity of $u$ on $R_{s}$, we have that $N_{R_{\varepsilon}}^{0}$ is a continuous function vanishing outside of $G$. Therefore, $G_{0}=\left\{N_{R_{\varepsilon}}^{0}>\lambda\right\}$ is an open set whose closure is contained in $G$.

Let $f$ be the characteristic function of the set $\left\{A_{R_{s}}>\gamma \lambda\right\}$ where $\gamma$ is a positive number to be chosen later. Here, let $f^{*}$ be the maximal function of $f$ defined by

$$
f^{*}(x)=\sup _{x \in B} \int_{B} f(s) d s / m(B)
$$

where $B$ is any ball containing $x$. Let

$$
E=\left\{D_{R_{\varepsilon}}^{0} \geqslant \beta \lambda, f^{*} \leqslant \frac{1}{2}, D_{R} \leqslant \delta \lambda\right\}
$$


where $\delta$ is a positive number to be chosen later. Assume that (33) holds. Then

$$
m\left(G_{0}\right) \leqslant \alpha m(E)+\alpha m\left(f^{*}>\frac{1}{2}\right)+\alpha m\left(D_{R_{\varepsilon}}>\delta \lambda\right) .
$$

By the Hardy-Littlewood maximal theorem,

$$
\frac{1}{2} m\left(f^{*}>\frac{1}{2}\right) \leqslant c\|f\|_{1}=c m\left(A_{R_{\varepsilon}}>\gamma \lambda\right)
$$

where the choice of $\theta$ depends only on $n$. Therefore, if

$$
\alpha m(E) \leqslant \frac{1}{2} m\left(G_{0}\right)
$$

holds for suitable $\gamma$ and $\delta$, the choice of which depends only on $\alpha, \beta$, and $n$, then the desired inequality also holds:

$$
m\left(G_{0}\right) \leqslant c m\left(A_{R_{\varepsilon}}>\gamma \lambda\right)+2 \alpha m\left(D_{R_{\varepsilon}}>\delta \lambda\right) .
$$

So the key step is to prove (34). Suppose, on the contrary, that $m\left(G_{0}\right)<2 \alpha m(E)$. Then, by Lemma 4 , there is a ball $B \subset G_{0}$, with at least one of its boundary points not in $G_{0}$, such that

$$
m(B) \leqslant \alpha_{0} m(E \cap B),
$$

where $a_{0}=2 \alpha e_{(25)}$. Without loss of generality, assume that $B$ is centered at the origin and has unit radius. Let $V$ be the interior of the cone with base $B$ and vertex at $(0,1)$. Then the closure of $V_{\varepsilon}=\{(x, y) \epsilon V: y>\varepsilon\}$ is contained in $R$. Choose $0<\eta<\frac{1}{2}$ so that the ball $B_{0}$ with center at the origin and radius $1-2 \eta$ satisfies $m\left(B_{0}\right) / m(B)=1-\left(2 \alpha_{0}\right)^{-1}$. Then, letting $E_{0}=E \cap B_{0}$, we have that

$$
m(B) \leqslant 2 \alpha_{0} m\left(E_{0}\right) .
$$

We need to consider several domains $W \subset W_{0} \subset W_{1}$. Let

$$
W_{i}=\bigcup_{x \in E_{i}} \Gamma(x) \cap V_{\varepsilon}, \quad i=0,1,
$$

where $E_{1}=\left\{f^{*} \leqslant \frac{1}{2}\right\} \cap B$. Notice first that

$$
\int_{E_{2}} A_{W_{1}}^{2}(x) d x \leqslant \gamma^{2} \lambda^{2} m(B),
$$

where $E_{2}=\left\{A_{R_{\varepsilon}} \leqslant \gamma \lambda\right\} \cap B$, since $A_{W_{1}} \leqslant A_{R_{\varepsilon}}$. Let $B(s, y)=\{x:|x-s|$ $<y\}$. Then, by Fubini's theorem,

$$
\int_{E_{2}} A_{W_{1}}^{2}(x) d x=\iint_{W_{1}} y^{1-n}|\nabla u(s, y)|^{2} m\left(E_{2} \cap B(s, y)\right) d s d y .
$$

We can get a lower bound for this integral as follows. Suppose that $(s, y) \in W_{1}$, Then, for some $x \in E_{1},(s, y) \in \Gamma(x) \cap V_{\varepsilon}$. Hence, $x \in B(s, y)$
and $f^{*}(x) \leqslant \frac{1}{2}$ so that

$$
m\left(A_{R_{\varepsilon}}>\gamma \lambda, B(s, y)\right) \leqslant \frac{1}{2} m(B(s, y))
$$

by the definition of $f^{*}$. Since $B(s, y) \subset B$, this implies that

$$
m\left(E_{2} \cap B(s, y)\right) \geqslant \frac{1}{2} m(B(s, y))=\frac{1}{2} v_{n} y^{n}
$$

where $v_{n}$ is the volume of a ball with unit radius. Combining these estimates, we obtain

$$
2 \gamma^{2} \lambda^{2} m(B) \geqslant v_{n} \iint_{W_{1}} y|\nabla u|^{2} d s d y
$$

and an even smaller integral if we replace $W_{1}$ by the smaller domain $W$, which we define below.

From now on, we assume that $u(0,1)=0$. This we can do without loss of generality since $N_{R_{\varepsilon}}^{0}$ and $\nabla u$ are unchanged if $u$ is replaced by $u-u(0,1)$. Using this assumption, we now show that

$$
N_{W_{0}}(x)>\frac{1}{2}(\beta-1) \lambda, x \in E_{0},
$$

for a suitable choice of $\delta$ depending only on $\alpha, \beta$, and $n$. We fix $x \in E_{0}$ and observe first that

where

$$
\beta \lambda \leqslant N_{R_{\varepsilon}}^{0}(x) \leqslant N_{W_{0}}^{0}(x)+N_{U_{1}}^{0}(x)+N_{U_{2}}^{0}(x)
$$

$$
\begin{aligned}
& U_{1}=\left\{(s, y) \epsilon R_{\varepsilon}:|s|<y-1\right\}, \\
& U_{2}=\left\{(s, y) \epsilon \Gamma(x) \cap R_{\varepsilon}:(s, y) \notin W_{0} \cup U_{1}\right\} .
\end{aligned}
$$

Note that the upper boundary of $U_{1}$ is part of the upper boundary of $R_{\varepsilon}$. We now use the fact that $B$ has a boundary point, say $x_{0}$, not in $G_{0}$ to obtain $U_{1} \subset \Gamma\left(x_{0}\right) \cap R_{\varepsilon}$ and

$$
N_{U_{1}}^{0}(x) \leqslant N_{R_{8}}^{0}\left(x_{0}\right) \leqslant \lambda
$$

If $(s, y) \in \Gamma(x) \cap R_{s}$, then

(38) $\quad y|\nabla u(s, y)| \leqslant \delta \lambda$

by the definition of $E_{0}$. We know by the mean value theorem that if (38) is satisfied for every point $(s, y)$ on the line segment joining the points $\left(s_{1}, y_{1}\right)$ and $\left(s_{2}, y_{2}\right)$ in $\boldsymbol{R}_{+}^{n+1}$, then

(39) $\quad\left|u\left(s_{1}, y_{1}\right)-u\left(s_{2}, y_{2}\right)\right| \leqslant \delta \lambda\left(\left|s_{1}-s_{2}\right|^{2}+\left(y_{1}-y_{2}\right)^{2}\right)^{\frac{1}{2}} / y_{1} \wedge y_{2}$.

Here $y_{1} \wedge y_{2}$ denotes the minimum of $y_{1}$ and $y_{2}$. Now let $\left(s, y_{1}\right)$ and $\left(s, y_{2}\right)$ belong to $U_{2}$. Then $|s| \geqslant y_{i}-1$; otherwise $\left(s, y_{i}\right)$ would belong to $U_{1}$. Also, $|x-s|<y_{i}$, implying that $|s|<y_{i}+|x|<y_{i}+1$. Therefore

$$
|s|-1 \leqslant y_{i} \leqslant|s|+1, \quad i=1,2
$$

so that $\left|y_{1}-y_{2}\right| \leqslant 2$. Using the fact that $x \in E_{0} \subset B_{0}$, we obtain $y_{1} \wedge y_{2}>\eta$. Therefore, by (39), we have

$$
N_{U_{2}}^{0}(x) \leqslant 2 \delta \lambda \eta^{-1}
$$


Using our assumption that $u(0,1)=0$, we also have

$$
|u(s, y)|=|u(s, y)-u(0,1)| \leqslant 2 \delta \lambda \eta^{-1}
$$

for all upper boundary points $(s, y)$ of $W_{0}$; this gives

$$
N_{W_{0}}^{0}(x) \leqslant N_{W_{0}}(x)+2 \delta \lambda \eta^{-1}
$$

Combining these estimates, we obtain (37) for all positive $\delta$ satisfying

$$
\delta<2^{-3} \eta(\beta-1) \text {. }
$$

We can now define $W$. Let $\Gamma(x, t)$ denote the cone $\Gamma(x)$ translated upward by $t$ units; that is,

$$
\Gamma(x, t)=\{(s, y):|x-s|<y-t\}
$$

If $(x, t) \epsilon W_{0}$, let

$$
N(x, t)=\sup _{(s, y) \in \Gamma(x, t) \cap W_{0}}|u(s, y)| .
$$

Finally, let $\theta=\frac{1}{2}(\beta-1)$ and

$$
W=\left\{(x, t) \in W_{0}: N(x, t)<\theta \lambda\right\} .
$$

By continuity and the fact that $u(0,1)=0, W$ is a nonempty open set. If $(x, t) \in W$, let $\left(x, t_{x}\right)$ be the point on the lower boundary of $W$ directly below $(x, t)$ :

$$
t_{x}=\inf \{t:(x, t) \epsilon W\}
$$

Then $\left|t_{x}-t_{s}\right| \leqslant|x-s|$ for all $x$ and $s$ in the projection $P_{W}$ of $W$ on $\boldsymbol{R}^{n}$. For $\delta$ satisfying (41), which we assume, the upper boundary of $W$ coincides with the upper boundary of $W_{0}$. Clearly, $W$ is a Lipschitz domain. By the definition of $W$,

$$
|u(s, y)| \leqslant \theta \lambda
$$

for all $(s, y) \epsilon W$, hence for all $(s, y) \epsilon \partial W$. Our next step is to show that, in a sense, $|u|$ is near $\theta \lambda$ on a large part of the lower boundary of $W$. To be precise, we let

$$
S=\left\{\left(x, t_{x}\right):\left|u\left(x, t_{x}\right)\right|>\frac{1}{2} \theta \lambda\right\}
$$

and denote by $P_{S}$ the projection of $S$ on $\boldsymbol{R}^{n}$. Then

$$
m\left(P_{S}\right) \geqslant o m(B)
$$

with the choice of $c$ depending only on $\alpha$ and $n$. To prove this, we let $g$ be the characteristic function of $P_{S}$ and $g^{*}$ be the maximal function of $g$ as defined in the proof of Lemma 2. Then

$$
E_{0} \subset\left\{g^{*} \geqslant \xi\right\}
$$

for a positive number $\xi$ whose choice depends only on $\eta$ and $n$, hence only on $\alpha$ and $n$. Inequality (43) easily follows from (44) and (35) since, by the Hardy-Littlewood theorem,

$$
\xi m\left(E_{0}\right) \leqslant \xi m\left(g^{*} \geqslant \xi\right) \leqslant c\|g\|_{I}=c m\left(P_{S}\right) .
$$

We now prove (44). Suppose that $x_{0} \in E_{0}$. Then, by (37), $N_{W_{0}}\left(x_{0}\right)>\theta \lambda$, so there is a point $(s, y) \in \Gamma\left(x_{0}\right) \cap W_{0}$ such that $|u(s, y)|>\theta \lambda$. Let $\left(x, t_{x}\right)$ be a point on the lower boundary of $W$, in $\Gamma(s, y) \cap W_{0}$, and satisfying $\left|u\left(x, t_{x}\right)\right|=\theta \lambda$. By (40) and (41), such a point exists. If $x=x_{0}$, we have $g^{*}\left(x_{0}\right)=1$; if $x \neq x_{0}$, we proceed as follows. By (39), (41), and the fact that $u(0,1)=0$, we have $\Gamma\left(x_{0}\right) \cap V_{\eta} \subset W$. Therefore, $B\left(x_{0}, \eta\right) \subset P_{W}$, $\left|x-x_{0}\right|<t_{x}<\eta$, and

Let $s$ be any point in

$$
B\left(x_{0},\left|x-x_{0}\right|\right) \subset P_{W} .
$$

$$
B\left(x,(1-\eta)\left|x-x_{0}\right|\right) \cap B\left(x_{0},\left|x-x_{0}\right|\right) \text {. }
$$

Then $\left|t_{x}-t_{s}\right| \leqslant|x-s|<(1-\eta)\left|x-x_{0}\right|<\left|x-x_{0}\right|$,

$$
t_{s} \geqslant t_{x}-|x-s|>\left|x-x_{0}\right|-(1-\eta)\left|x-x_{0}\right|=\eta\left|x-x_{0}\right|
$$

and, using (39) once more, we obtain

$$
\begin{aligned}
\theta \lambda-\left|u\left(s, t_{s}\right)\right| & \leqslant\left|u\left(x, t_{x}\right)-u\left(s, t_{s}\right)\right| \\
& \leqslant \delta \lambda\left(|x-s|^{2}+\left(t_{x}-t_{s}\right)^{2}\right)^{\frac{1}{s}} / t_{x} \wedge t_{s} \\
& \leqslant 2 \delta \lambda \eta^{-1} .
\end{aligned}
$$

So, by (41), we have $\left|u\left(s, t_{s}\right)\right|>\frac{1}{2} \theta \lambda$ and $s$ is in $P_{S}$. Therefore,

where

$$
g^{*}\left(x_{0}\right) \geqslant m\left(P_{S} \cap B\left(x_{0},\left|x-x_{0}\right|\right)\right) / m\left(B\left(x_{0},\left|x-x_{0}\right|\right)\right) \geqslant \xi
$$

$$
\xi=m\left(B\left(x,(1-\eta)\left|x-x_{0}\right|\right) \cap B\left(x_{0},\left|x-x_{0}\right|\right)\right) / m\left(B\left(x_{0},\left|x-x_{0}\right|\right)\right),
$$

which clearly depends only on $\eta$ and $n$. This completes the proof of (44).

Keeping the same meaning for $\partial / \partial n$ and $\sigma$, we now apply Green's theorem with the same proviso as in Section 5. Denote the lower boundary of $W$ by $(\partial W)^{-}$and the upper boundary by $(\partial W)^{+}$. We have $\partial y / \partial n \geqslant 2^{-\frac{1}{2}}$ on $(\partial W)^{-}, \sigma(S) \geqslant m\left(P_{S}\right)$, and $\sigma(\partial W) \leqslant 4 m(B)$. Using $(38),(40),(42)$, and (43), we obtain

$$
\begin{aligned}
2 \int_{W} y|\nabla u|^{2} d s d y & =\int_{W} y \Delta u^{2} d s d y \\
& =\int_{(\partial W)^{-}} u^{2} \frac{\partial y}{\partial n} d \sigma+\int_{(\partial W)^{+}} u^{2} \frac{\partial y}{\partial n} d \sigma-2 \int_{\partial W} y \frac{\partial u}{\partial n} u d \sigma \\
& \geqslant \int_{S} 2^{-3} \theta^{2} \lambda^{2} d \sigma-\int_{(\partial W)^{+}}\left(2 \delta \lambda \eta^{-1}\right)^{2} d \sigma-2 \int_{\partial W} \delta \theta \lambda^{2} d \sigma \\
& \geqslant \lambda^{2} m(B)\left[c_{(43)^{2}} 2^{-3} \theta^{2}-16 \delta^{2} \eta^{-2}-8 \delta \theta\right]
\end{aligned}
$$


By (36), we have

$$
4 \gamma^{2} \geqslant v_{n}\left[c_{(43)} 2^{-3} \theta^{2}-16 \delta^{2} \eta^{-2}-8 \delta \theta\right],
$$

and we see that this leads to a contradiction if both $\delta$ and $\gamma$ are chosen suitably small. Therefore, (34) must hold and the proof of Theorem 3 is complete.

\section{References}

[1] D. L. Burkholder and R. F. Gundy, Extrapolation and interpolation of quasi-linear operators on martingales, Acta Math. 124 (1970), pp. 249-304.

[2] -, - and M. L. Silverstein, A maximal function characterization of the class $H^{p}$, Trans. Amer. Math. Soc. 157 (1971), pp. 137-153.

[3] A. P. Calderón, On the behavior of harmonic funotions at the boundary, Trans. Amer. Math. Soc. 68 (1950), pp. 47-54.

[4] - On a theorem of Marcinkiewicz and Zygmund, Trans. Amer. Math. Soc. 68 (1950), pp. 55-61.

[5] - Commutators of singular integral operators, Proc. Nat. Acad. Sci. 53 (1965), pp. 1092-1099.

[6] C. Fefferman and E. M. Stein, $H^{p}$-spaces of several variables, Acta Math., to appear.

[7] G. Gasper, On the Littlewood-Paley and Lusin functions in higher dimensions, Proc. Nat. Acad. Sci. 57 (1967), pp. 25-28.

[8] J. Horváth, Sur les fonctions conjuguées à plusieurs variables, Nederl. Akad. Wetensch. Prac. Ser. A. $56=$ Indagationes Math. 15 (1953), pp. 17-29.

[9] J. Marcinkiewicz and A. Zygmund, A theorem of Lusin, Duke Math. J. 4 (1938), pp. $473-485$.

[10] C. Segovia, On the area function of Lusin, Studia Math 33 (1969), pp 311-343.

[11] D. C. Spencer, A function-theoretio identity, Amer. J. Math. 65 (1943), pp. 147160.

[12] E. M. Stein, On the functions of Littlewood-Paley, Lüsin, and Marcinliewiez, Trans. Amer. Math. Soc. 88 (1958), pp. 430-466.

[13] - On the theory of harmonic functions of several variables II. Behavior near the boundary, Acta Math. 106 (1961), pp. 137-174.

[14] - Singular integrals and differentiability properties of functions, Princeton 1970.

[15] - and G. Weiss, On the theory of harmonio functions of several variables $I$. The theory of $B^{p}$-spaces, Acta Math. 103 (1960), pp. 25-62.

\section{An inequality for the indefinite integral of a function in $L^{q}$}

by

MAX JODEIT, Jr* (Chicago, Ill.)

To A. Zygmund, real analyst

Abstract. Let $p, q$ be positive numbers with $1=1 / p+1 / q$. Let $F$ be continuously differentiable on the positive reals and zero at the origin. Let $g$ denote the $p$ th power of $|F|$. J. Moser has shown that if $q$ is at least 2 and the $q$ norm of $F^{\prime}$ is at most 1 then the integral of $\exp (g(x)-x)$ is bounded by a constant depending only on $q$. A new proof of this is given, and the result extended to all $q>1$.

In his paper "A sharp form of an inequality by N. Trudinger", J. Moser ([2], Theorem 1) proves that if $D$ is a bounded domain in $R^{n}$, $n \geqslant 2$, and $u$ is a $C^{1}$ function with compact support in $D$ such that $\int_{D}|\operatorname{grad} u(x)|^{n} d x \leqslant 1$ then $\int_{D} \exp \alpha_{n}|u(x)|^{n /(n-1)} d x \leqslant c_{n}$ for certain constants $\alpha_{n}, c_{n}$ independent of $u$.

Earlier N. Trudinger [3] proved this for some $\alpha>0$.

Moser elegantly reduces the question to the following one-dimensional inequality which he proves for $q \geqslant 2$.

The present paper contains a new proof which incidentally works for $q>1$.

THEOREM 1.1. Let $q$ and $p$ denote positive numbers with $1<q<\infty$, $\frac{1}{p}+\frac{1}{q}=1$ (Hölder conjugates). Let $f$ be Lebesgue measurable on $(0, \infty)$, let $\int_{0}^{\infty}|f(x)|^{q} d x \leqslant 1$, and let $F^{\prime}(x)=\int_{0}^{x} f(t) d t$. Then there exists a number $C_{q}$ depending only on $q$ such that

$$
\int_{0}^{\infty} e^{|F(x)|^{p}} e^{-x} d x \leqslant C_{q} .
$$

In what follows we consider only non-negative functions $f$. An equivalent theorem arises through use of the substitution $x=\log \frac{1}{u}$ :

* Partially supported by the National Science Foundation under NSF grant GP 28271. 